\title{
Identification, tissue specific expression, and chromosomal localisation of several human dynein heavy chain genes
}

\author{
Amit K Maiti ${ }^{1}$, Marie-Geneviève Mattéi ${ }^{2}$, Mark Jorissen $^{3}$, Armin Volz ${ }^{4}$, Andreas Zeigler ${ }^{4}$ \\ and Patrice Bouvagnet ${ }^{5}$
}

\begin{abstract}
${ }^{1}$ Division of Medical Genetics, Geneva University School of Medicine, Geneva, Switzerland; ${ }^{2}$ IN SERM U 406, Faculté de M édecine, Marseille, France; ${ }^{3}$ Centrum voor Menselijke Erfelijkheid, Campus Gasthuisberg, $O \& N$, Leuven, Belgium; ${ }^{4}$ Institut für Immungenetik, Universitätsklinikum Charité, Humboldt-Universität zu Berlin, Berlin, Germany; ${ }^{5}$ Laboratoire de Génétique M oléculaire Humaine, Faculté de Pharmacie, Lyon, France
\end{abstract}

Sliding between adjacent microtubules within the axonema gives rise to the motility of cilia and flagella. The driving force is produced by dynein complexes which are mainly composed of the axonemal dynein heavy chains. We used cells of human respiratory epithelium after in vitro ciliogenesis to clone cDNA fragments of nine dynein heavy chain genes, one of which had never been identified before. Dynein heavy chains are highly conserved from protozoa to human and the evolutionary ancestry of these dynein heavy chain CDNA fragments was deduced by phylogenetic analysis. These dynein heavy chain cDNAs are highly transcribed in human tissues containing axonema such as trachea, testis and brain, but not in adult heart or placenta. PAC clones containing dynein heavy chains were obtained and used to determine by FISH their chromosomal position in the human genome. They were mapped to 2p12-p11, 2q33, 3p21.2-p21.1, $13 q 14,16 p 12$ and 17p12. The chromosomal assignment of these dynein heavy chain genes which was confirmed by GeneBridge 4 radiation hybrid screening, will be extremely useful for linkage analysis efforts in patients with primary ciliary dyskinesia (PCD). European Journal of Human Genetics (2000) 8, 923-932.

Keywords: chromosomal mapping; human; dynein; heavy chain; primary ciliary dyskinesia; evolution

\begin{abstract}
Introduction
Immotile cilia syndrome (ICS) or primary ciliary dyskinesia (PCD) is an autosomal recessive disorder in humans impairing the beating of cilia and flagella. Ciliated epithelial cell lining is present in respiratory tract, paranasal sinuses, middle ear, efferent duct of testis, Fallopian tubes, brain and spinal cord. Patients with PCD have recurrent respiratory tract infections leading to bronchietactasis and often male infertility. About $50 \%$ of the patients have situs inversus and hence Kartagener syndrome. The motility of cilia and flagella is generated in the axoneme which is composed of more than 250 different proteins. ${ }^{1}$ Electron microscopic studies on the
\end{abstract}

Correspondence: Patrice Bouvagnet, Laboratoire de Génétique Moléculaire Humaine, Faculté de Pharmacie, Université $\mathrm{Cl}$. Bernard Lyon 1, 8 av. Rockefeller, F-69373, Lyon, Cedex 08, France.

Tel: +33 4787771 21; Fax: +33 4787775 68;

E-mail: pbouv@rockefeller.univ-lyon1.fr

Received 17 January 2000; revised 11 July 2000; accepted 14 July 2000 ultrastructure of cilia in PCD patients revealed frequent abnormalities in the inner, outer or both dynein arms and in the central pair of microtubules. ${ }^{2}$ Dynein is one of the major components of the axoneme. The A-tubule of the microtubular doublets is connected to outer and inner dynein arms at a regular distance and each dynein arm consists presumably of two to three heavy chains, two to four intermediate chains and several light chains. ${ }^{3}$ Each dynein heavy chain molecule has a highly conserved putative ATP-binding motif (P-loop) suggesting a force generation during movement. The function of light and intermediate chains is poorly understood. They may have an important role in regulation, assembly of dynein complex and attachment of heavy chains to the A-tubule. ${ }^{4,5}$ Recently, DNAI1, a dynein intermediate chain was implied in PCD since a patient with PCD was found to be a double heterozygote for mutations in this gene. ${ }^{6}$ 
Numerous distinct dynein heavy chain genes have been isolated from Paramecium, ${ }^{7}$ Chlamydomonas, ${ }^{8,9}$ sea urchin, ${ }^{10}$ rat, ${ }^{11,12}$ mouse, ${ }^{13}$ and human. ${ }^{13-16}$ However, comparing the dynein heavy chain gene family in well characterised species such as sea urchin suggests that either mammals have less axonemal dynein heavy genes or that some mammal genes are still to be discovered.

As a first step, in vitro ciliogenesis of the ciliated epithelial cells (obtained by bronchial or nasal biopsy of patients) was obtained after sequential monolayer and suspension culture. ${ }^{17,18}$ From the regenerated ciliated epithelial cells, we cloned and identified several dynein heavy chain cDNAs, one of which corresponds to a new human dynein heavy chain. The transcription of these dynein heavy chain cDNAs was studied in different human tissues including trachea, brain, testis, placenta and heart. Five of these genes and one pseudogene werelocalised in chromosomes by in situ fluorescence hybridisation (FISH) and by radiation hybrid mapping.

\section{Materials and methods \\ Human epithelial cell culture}

Human nasal epithelial cells were treated in a sequential monolayer suspension culture system described previously. ${ }^{17,18}$ After about 4 weeks, stable aggregates, spheroids and vesicles were formed of mature epithelial cells with functional ciliary beating.

\section{Primer design, RT-PCR amplification, cloning and sequencing of dynein heavy chain genes}

Primers were designed around the first P-loop region according to amino acid sequences of already published dynein heavy chain genes; DNAHIoop-1F,-2F,-3F,-4R (Table 1). During the course of this study, Neesen et al $^{13}$ reported several dynein heavy chain isoform from human testis. A primer pair was selected from their hdhc9 sequence: RT-DNAH5F and -5R (Table 1).

Total RNA was isolated from the cultured cells as already described. ${ }^{19,20}$ RT-PCR was carried out with GeneAmp RT-PCR Kit (Perkin Elmer, Essonne, France) with $2 \mu \mathrm{g}$ of RNA. PCR products were cloned in PCR2.1 vector of the TA-cloning Kit (Invitrogen, Gronigen, The Netherlands). White colonies were checked for correct insert size by either restriction digestion or by PCR with the parent primers. Clones were sequenced and sequences were screened with BLAST. Each clone with a strong homology to dynein heavy chain genes was sequenced again in both directions. Amino acid sequences were deduced and all sequences were aligned to make comparison among them.

After several reports, the nomenclature system of dynein heavy chain genes by different groups is confusing and the homologue of each isoform in mammals and in human is difficult to follow. Here, we followed the nomenclature suggested by the HUGO Nomenclature Committee for naming genes. The name for human axonemal dynein heavy chain gene is DNAH (DN for dynein, A for axonal and $\mathrm{H}$ for heavy chain). A prefix in parenthesis is given to specify species: HSA for Homo sapiens, RNO for Rattus norvigecus and

Table 1 Primers used in this study

\begin{tabular}{|c|c|c|c|}
\hline $\begin{array}{l}\text { DNAHL oop - 1F } \\
\text { DNAHL o op - } 3 \mathrm{~F}\end{array}$ & $\begin{array}{l}\text { TAYGGNTTYGARTAYYTNGG } \\
\text { ACNGGNAARACNGARACNAC }\end{array}$ & $\begin{array}{l}\text { DNAHL o op - } 2 \mathrm{~F} \\
\text { DNAHL o op - } 4 \mathrm{R}\end{array}$ & $\begin{array}{l}\text { GTNCRRACNNNCYTNACNGA } \\
\text { CCNGGRTTCATNGTDATRAA }\end{array}$ \\
\hline RT - DNAH1F & AACTGAGACCACCAAAGAC & RT-DNAH1R & CCTTCTGGATGGTGGTGATC \\
\hline RT - DNAH3F & GCAAGACAGAAACCACCAAAGATT & RT-DNAH3R & ATGGCTTGTTGGATGCTGAGG \\
\hline RT - DNAH5 F & CACAAAAGACATGGGAAG & RT-DNAH5R & TGCTGCCACTGATAATAC \\
\hline RT - DNAH6F & GCAAAAGCTCTTGCCATCCAGT & RT - DNAH6R & CATCACCAACTTTATTTCCC \\
\hline RT - DNAH7F & GATGGGTTGGATTATTTGG & RT - DNAH7R & TGGGGTCAAGTTTTAGTTC \\
\hline RT - DNAH9F & ATCCTGGTCTATGTGTTC & RT-DNAH9R & AGATACCGACAGAAGGATTC \\
\hline RT - DNAH1 OF & TGGGGAAGATTTTCTCTGG & RT - DNAH1OR & CGGGTTCATTGTGATAAAG \\
\hline $\mathrm{RT}-\mathrm{DNAH} 11 \mathrm{~F}$ & CGTGCCCTTGGCATGATGGTCTAT & $\mathrm{RT}-\mathrm{DNAH} 11 \mathrm{R}$ & TCCAACTGATGGCTTGAGTGTG \\
\hline DNAH - $2 \mathrm{~F}$ & GAAGAGCCTAATTTCTCC & $\mathrm{DNAH}-1 \mathrm{Rb}$ is & ATCTCCACACCCTCAAAC \\
\hline DNAH - 6Fbis & GTGTGTGGTCTTTACTG & & \\
\hline DNAH - $9 i F$ & GGATGGAGAATGGCTTCAAC & DNAH - $9 i \mathrm{R}$ & CAAGAGGACATAGACTTCCGC \\
\hline DNAH - $12 \mathrm{~F}$ & GACTTGGCTAAAGCTCTTGCTGTA & & \\
\hline DNAH - $14 \mathrm{~F}$ & CCTTAGGCAAACATTGTG & & \\
\hline $\mathrm{RH}-\mathrm{DNAH} 1 \mathrm{~F}$ & TGGGCTCTGAACACATGTGC & RH-DNAH1R & GTGAGTATGGCAGATTTGAGG \\
\hline $\mathrm{RH}-\mathrm{DNAH} 3 \mathrm{~F}$ & AGTGTGTGGGTATCATTCAGG & $\mathrm{RH}-\mathrm{DNAH} 3 \mathrm{R}$ & CTTGGCTAAGCAGGTAAGC \\
\hline $\mathrm{RH}$ - DNAH6F & AACATGAGTGCAGTGCCAGC & RH - DNAH6R & AGAGCTGCTGGAGAAGCTTG \\
\hline RH - DNAH7F & CTTGTCAGTCCATGAGCTTC & RH - DNAH7R & CTCGCAACACACAATCTACC \\
\hline RH - DNAH1 OF & TCCAATCTTTGGTAGACACC & RH - DNAH1OR & TTACAGAGGCAGGTGTGACC \\
\hline
\end{tabular}

DNAHLoop primers are degenerated primers used to clone dynein CDNA; RT-DNAH primers were used for specific RT-PCR amplifications; RH-DNAH primers were used for radiation hybrid mapping. 
Table 2 Dynein nomenclature

\begin{tabular}{|c|c|c|c|c|c|c|c|}
\hline $\begin{array}{l}\text { Maiti } \\
\text { et al } \\
\text { Human }\end{array}$ & $\begin{array}{l}\text { Vaughan } \\
\text { et al }{ }^{14} \\
\text { Human }\end{array}$ & $\begin{array}{l}\text { Chapelin } \\
\text { et al }{ }^{16} \\
\text { Human }\end{array}$ & $\begin{array}{l}\text { Neesen } \\
\text { et al }{ }^{13} \\
\text { Human }\end{array}$ & $\begin{array}{l}\text { Tanaka } \\
\text { et al }{ }^{11} \\
\text { Rat }\end{array}$ & $\begin{array}{l}\text { Gibbons } \\
\text { et al }{ }^{10} \\
\text { Sea urchin }\end{array}$ & $\begin{array}{l}\text { HUGO } \\
\text { in }\end{array}$ & $\begin{array}{l}\text { Chrom. } \\
\text { position } \\
\text { Human }\end{array}$ \\
\hline DNAH1 & Dnahc1 & Dnahcl & hdhc7 & Dlp1 & Tgdyh6 & DNAHI & $3 p 21-21.3$ \\
\hline- & Dnahc2 & Dnahc2 & - & Dlp2 & Tgdyh5c & DNAH2 & \\
\hline DNAH3 & Dnahc3 & Dnahc3b & hdhc8 & Dlp3 & Tgdyh7b & DNAH3 & $16 p 12$ \\
\hline DNAH5 & Dnahc5 & - & - & Dlp5 & Tgdyh3b & DNAH5 & \\
\hline DNAH6 & Dnahc6 & - & - & Dlp6 & Tgdyh5a & DNAH6 & $2 \mathrm{p} 12$ \\
\hline DNAH7 & Dnahc7 & - & hdhc2 & Dlp7 & Tgdyh7a & DNAH7 & \\
\hline DNAH8 & Dnahc8 & - & hdhc9 & DIp8 & Tgdyh3a & DNAH8 & $6 p 21$ \\
\hline DNAH9 & Dnahc9 & - & hdhcl & Dlp9a & Tgdyh2 & $\begin{array}{l}\text { DNAH9/ } \\
\text { DNAH17L }\end{array}$ & $17 p 12$ \\
\hline DNAH10 & - & - & - & Dlp10 & Tgdyh4 & - & $13 q 14$ \\
\hline DNAH11 & Dnahc11 & Dnahc11 & hdhc4 & Dlp11 & Tgdyh1 & DNAH11 & $7 p 15$ \\
\hline- & Dnahc12 & Dnahc3 & - & DIp12 & Tgdyh7c & DNAH12 & \\
\hline- & Dnahc14 & - & - & - & Tgdyh5b & DNAH14 & \\
\hline $\begin{array}{l}\text { Pseudogene } \\
\text { of DNAH7 } \\
\text { (DNAH7p) }\end{array}$ & - & - & - & - & - & - & $2 q 33$ \\
\hline
\end{tabular}

athis study; note: assignment of isoform with sea urchin and rat was done by amino acid identity in the P-loop region of amino acid 1 to 101 of Figure 1; HUGO-NC: current HUGO nomenclature.

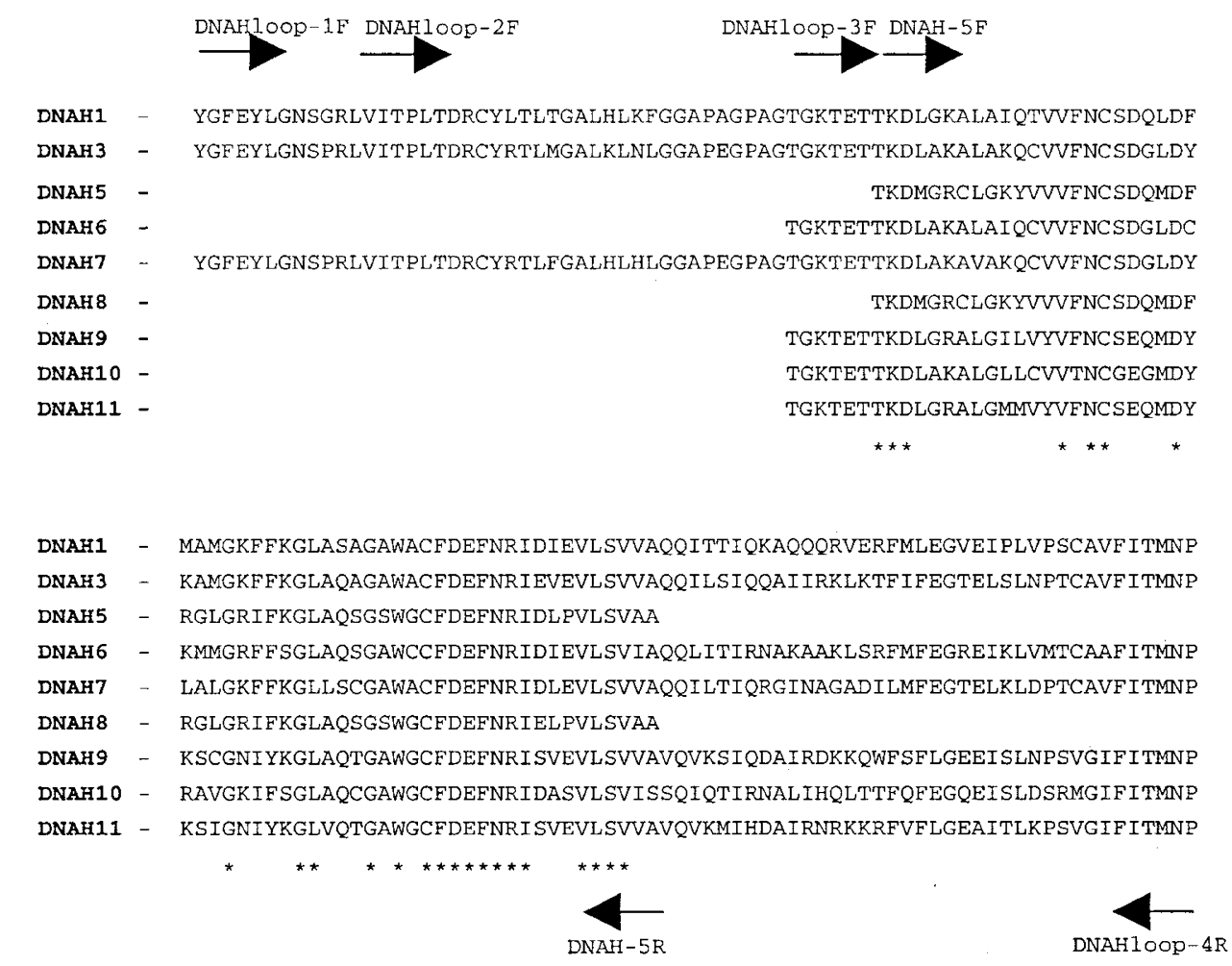

Figure 1 Alignment of the deduced amino sequences and position of the different primers for picking these cDNAs around the P-loop region of the dynein heavy chain gene. This part of the gene is highly conserved. DNAHloop-1F, DNAHloop-2F, DNAHloop-3F and DNAH-5F are sense strand primers. DNAHloop-4R and DNAH-5R are antisense primers. All dynein heavy chain cDNAs were cloned with primers 1, 2, 3, 4 except DNAH5 and -8 which were cloned with specific non-degenerated primers DNAH-5F and DNAH-5R. Arrows indicate the positions of primers, see Materials and methods for sequence: * indicates the conserved amino acids in all represented genes. 
TGR for Tripneustes gratilla. In rat and human, these names bear Arabic numerals for each dynein heavy chain gene according to Tanaka et al ${ }^{11}$ and Vaughan et al. ${ }^{14}$ In other organisms each dynein gene bears its original identifying number and letter, as for example, Tripneustes gratilla DYH5a becomes (TGR)DNAH5a. Table 2 summarises old and new names.

\section{Phylogenic analysis of dynein heavy chain genes}

Amino acid sequences of sea urchin, rat, mouse and human sequences were taken from databases and aligned by CLUSTALW. The phylogenetic tree of the dynein heavy chain genes were constructed by the program PHYLIP in neighbour-joining method with Poisson correction, global gap removal and 1000 bootstrap replicates. ${ }^{21}$ Forty-five amino acid positions for each clone were taken into account.

\section{Expression of dynein heavy chain genes in different tissues}

For axonemal dynein heavy chain gene expression, $2 \mu \mathrm{g}$ of total RNA from human brain, testis and trachea (Invitrogen) and from adult heart was reverse transcribed with random hexamer as specified in Perkin-Elmer RT-PCT Kit. An aliquot of the reaction was further PCR amplified with isoform specific primers (Table 1$)$. Annealing temperatures were: DNAH1 $\left(54^{\circ} \mathrm{C}\right),-3\left(56^{\circ} \mathrm{C}\right),-5\left(55^{\circ} \mathrm{C}\right),-6\left(52^{\circ} \mathrm{C}\right),-7\left(53^{\circ} \mathrm{C}\right),-9$ $\left(56^{\circ} \mathrm{C}\right),-10\left(53^{\circ} \mathrm{C}\right)$ and $-11\left(58^{\circ} \mathrm{C}\right)$.

\section{Hybridisation of PAC library and identification of dynein heavy chain genes}

A clone of each dynein isoform was individually amplified and radiolabelled. Radioactive PCR products were then pooled to get the 'pooled dynein probe' which was used to screen PAC filters. Thirty positive PAC clones were obtained from Lawrence Livermore $\mathrm{N}$ ational Laboratory ${ }^{22}$ and checked again with the pooled dynein probe. Specific upstream primers were designed, pooled and used with DNAH pool-4R in an attempt to amplify dynein fragments for PAC clones. Upstream specific primers are: RT-DNAH1F; DNAH-2F, ${ }^{14}$ RT-DNAH3F, RT-DNAH5F, DNAH-6Fbis, RT-DNAH7F, RT-DNAH9F, RT-DNAH10F, RT-DNAH11F, RT-DNAH12F, ${ }^{16}$ RT-DNAH14F. ${ }^{14}$ Three PAC clones amplified a 276 bp fragment that after cloning and sequencing of several clones corresponded to (HSA)DNAH3. Primers derived from (HSA)DNAH1 cDNA sequence were used for PCR amplification of each PAC clone DNA. Primers were RT-DNAH1F and DNAH-1Rbis. An amplimer product (1182 bp) was obtained from 5 PAC clones and sequenced. Their sequence identity with the (HSA)DNAH1 mRNA confirmed that these clones contained the (HSA)DNHA1 gene. Primers RT-DNHA7F and $-7 R$ amplified a 255 bp product from PAC P0764. Subsequent sequencing revealed a 54 bp intronic sequence included between two exons. We noted that the $5^{\prime}$ exonic sequence had an A insertion just before the normal GT donor site, suggesting that it might be a pseudogene.
(HSA)DNAH6 and (HSA)DNAH10 containing PACs were identified by HindIII restriction digestion, Southern blotting and hybridisation with the pooled dynein probe. The $1.1 \mathrm{~kb}$ (DNAH6) and $2.1 \mathrm{~kb}$ (DNAH10) positive bands were purified, cloned, and sequences were obtained with respective genespecific upstream primer (RT-DNAH6R and RT-DNAH10F). When (HSA)DNAH 9 sequence was subjected to BLAST search, it gave a strong homology to some portion of a genomic fragment (EMBL: AC005701). Base to base comparison demonstrated an intronic sequence between the two exonic sequences. Primers were designed from the intronic sequence: DNAH9i-F and -R (Table 1 and an amplification product of the expected size (249 bp) was obtained from PAC P18162. The sequence of this fragment was exactly homologous to the genomic fragment demonstrating that PAC P18162 contained the DNAH9 gene. We were unable to identify PAC clones that contained the p-loop region of (HSA)DNAH2, -5, -7, -8 and -11.

\section{Fluorescence in situ hybridisation of PAC}

FISH of PAC clones was done by standard protocol. PAC clone DNA was biotinylated by nick translation with biotin16-UTP, according to the manufacturer's protocol (Roche Diagnostics, Meylan, Isève, France). Hybridisation of chromosome spreads was performed according to standard protocol. ${ }^{23,24}$ A total of 20 metaphase cells was analysed for each PAC clone. PAC clones H2110, L1135, J1436, P0764, P18162, and L2158 were used to map DNAH1, 3, 6, 7 pseudogene, 9 and 10 , respectively.

\section{GeneBridge 4 radiation hybrid screening}

Radiation hybrids (GeneBridge 4) were obtained from HGMP-RC. Primer sequences were derived from PAC genome sequences (Table 1). Positives and negatives were scored with RHyME (Radiation Hybrid Mapping Environment) program with the 'all chromosomes' option.

\section{Results \\ Cloning, identification, and phylogeny of dynein heavy chain genes}

To identify the dynein heavy chain genes, we aligned the Chlamydomonas, sea urchin and rat dynein heavy chain genes from databases and designed for RT-PCR (Figure 1) four degenerated primers around the first P-loop region. Total cellular RNA was isolated from human regenerated ciliated epithelial cells and RT-PCR was performed with this set of primers by turn. The expected size bands were directly cloned in E. coli vector. Insert size was checked by PCR and sequenced. Then DNA sequence was analysed by BLAST to identify dynein heavy chain genes. Out of almost 400 sequenced clones, 62 showed a striking homology with dynein heavy chain genes. These 62 clones were further resolved in nine different isoforms (Figure 1). It is interesting that the primer pair DNAHloop-3F, DNAHloop-4R was highly 
dynein heavy chain specific since 25 out of 27 sequenced clones contained one of seven different dynein isoforms. Moreover, these seven dynein heavy chain isoforms contain the TGKTETT amino acid sequence specific to axonemal P-loop ${ }^{12}$ which suggests that they actually are axonemal dyneins. Nevertheless, this primer pair failed to amplify (HSA) DNAH5 and -8 which were only amplified by a pair of non-degenerated primers (RT-DNAH5F and -5R). ${ }^{13}$ These two dynein heavy chains differ only by one amino acid in this highly conserved region (Figure 1 ).

During the course of this study, Neesen et $\mathrm{al}^{13}$ and Chapelin et $\mathrm{al}^{16}$ reported several dynein heavy chain isoforms from human testis and genomic DNA. After comparison, we report here one new isoform (HSA)DNAH10 which had not been isolated in human.

A phylogenetic tree (Figure 2) was constructed by taking amino acid sequences of the dynein heavy chain genes of different organisms: sea urchin, mouse, rat, and human. In the evolutionary tree, cytoplasmic and axonemal dyneins are distinctly separated and their evolutionary origin can be traced. Putative outer and inner arm dyneins are distinctly clustered and in most cases, although not all, their respective homologues have been isolated in all these organisms. (TGR)DNCHIb is the sea urchin DYHIB which is homologous $^{25}$ to human DYH1B (DNCH2). Human cytoplasmic dynein (HSA)DNCH1 and (HSA)DNCH2 were isolated from non-axonema containing HELA cells ${ }^{15}$ and clustered with rat cytoplasmic homologues, (RNO)Dnch1 and (RNO)Dnah4, respectively. The later was isolated from rat brain but was assigned as a cytoplasmic dynein. ${ }^{11}$ (HSA)DNAH12 also was isolated from HELA cells although this isoform has the axonemal P-loop motif (TGKTETT) and according to the phylogenetic tree this dynein is more probably axonemal than cytoplasmic dynein. ${ }^{15}$ (HSA)DNAH10, the newly reported dynein heavy chain, clusters with (MMU)Dnah4, (RNO)Dnhal0 and (TGR)DNAH4 in the group of potential inner arm dyneins. (TGR)DNAH5A and (HSA)DNAH14 have no rat and mouse counterparts suggesting that these isoforms have not been yet cloned in these mammals.

\section{Expression of dynein heavy chain genes in different human tissues}

In our cloning strategy, we observed that 25 out of 27 clones arising from the RT-PCR product of a particular pair of primers (DNAHloop-3F and DNAHloop-4R) were dynein heavy chain genes and consisted of altogether seven different isoforms. Therefore, these primer pairs were regarded as axonemal dynein specific primers. To test the expression pattern of the dynein genes, we designed gene-specific primers (Table 1) and performed RT-PCR experiments for each dynein gene using RNA from brain, testis, trachea and heart. It can be deduced from Figure 3 that each gene is expressed in ciliated tissues but not in adult heart. The two bands that were amplified with RT-DNAH7F and -7R (Figure 3 ) were cloned and sequenced. The shorter band (201 bp) was

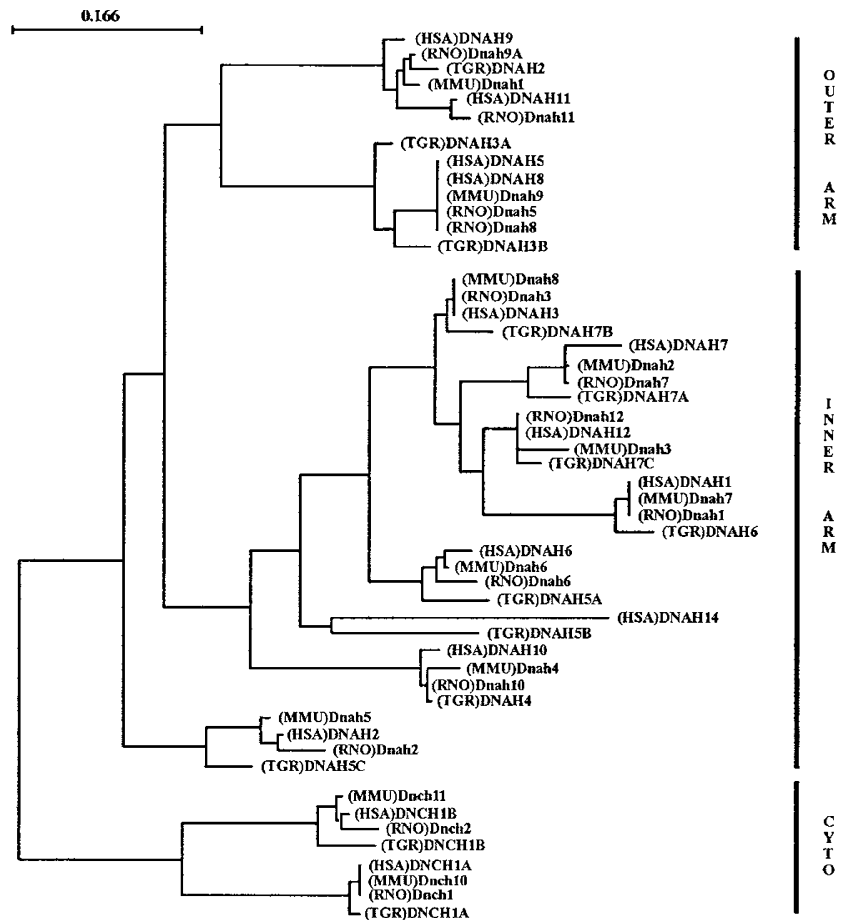

Figure 2 Phylogenetic tree of the dynein heavy chain amino acid sequences. Sequences of Tripneustes gratilla (TGR), Rattus norvegicus (RNO), M us musculus (MMU) and human (HSA) were obtained from EMBL and each gene is named according to the rules of the HUGO nomenclature committee: (HSA)DNAH1 (HSA1320833); (HSA)DNAH2 (U83570); (HSA)DNAH3 (HSA132085 and Z83805); (HSA)DNAH5 (HSA132090 and U61738); (HSA)DNAH6 (HSA132086 and U61736); (HSA)DNAH7 (HSA132084 and Z83801); (HSA)DNAH8 (HSA132091 and Z83806); (HSA)DNAH9 (HSA132088); (HSA)DNAH10 (HSA132089); (HSA)DNAH11 (HSA132087); (HSA)DN AH12 (Z83802); (HSA)DN AH114 (U61741); (HSA)DNCH1A (L23958) and (HSA)DNCH1B (HSU20552). (MMU)Dnah1 (MMMDHC1); (MMU)Dnah2 (MMMDHC2); (MMU)Dnah3 (MMDHC3); (MMU)Dnah4 (MMDHC4); (MMU)Dnah5 (MMDHC5); (MMU)Dnah6 (MMDHC6); (MMU)Dnah7 (MMMDHC7); (MMU)Dnah8 (MMMDHC8); (MMU)Dnah9 (MMMDHC9); (MMU)Dnch10 (MMMDHC10) and (MMU)Dnch11 (MMMDHC11). (RNO)Dnah1 (RNDLP1A); (RNO)Dnah2 (RNDLP2B); (RNO)Dnah3 (RNDLP3C); (RNO)Dnah5 (RNDLP5E); (RNO)Dnah6 (RNDLP6F); (RNO)Dnah7 (RNDLP7G and RN32182); (RNO)Dnah8 (RNDLP8H); (RNO)Dnah9A (RNDLP9I); (RNO)Dnah10 (RNDLP10K); (RNO)Dnah11 (RNDLP11L); (RNO)Dnah12 (RNDLP12M); (RNO)Dnch1 (RNU61742) and (RNO)Dnch2 (RNU61748). (TGR)DNAH2 (TGDYH2); (TGR)DNAH3A (TGYH3A); (TGR)DNAH3B (TGYH3B); (TGR)DNAH3C (TGYH3C); (TGR)DNAH4 (TGYH4); (TGR)DNAH5A (TGYH5A); (TGR)DNAH5B (TGYH5B); (TGR)DNAH5C (TGYH5C); (TGR)DNAH6 (TGYH6); (TGR)DNAH7A (TGYH7A); (TGR)DNAH7B (TGYN7B); (TGR)DNAH7C (TGYN7C); (TGR)DNAH7D (TGYN7D); (TGR)DNCH1A (TGDYH1A) and (TGR)DN CH1B (TGYH1B). The putative outer, inner arm and cytoplasmic dyneins are clustered in the tree. The scale of branch length indicates the mean number of residue changes per site. 


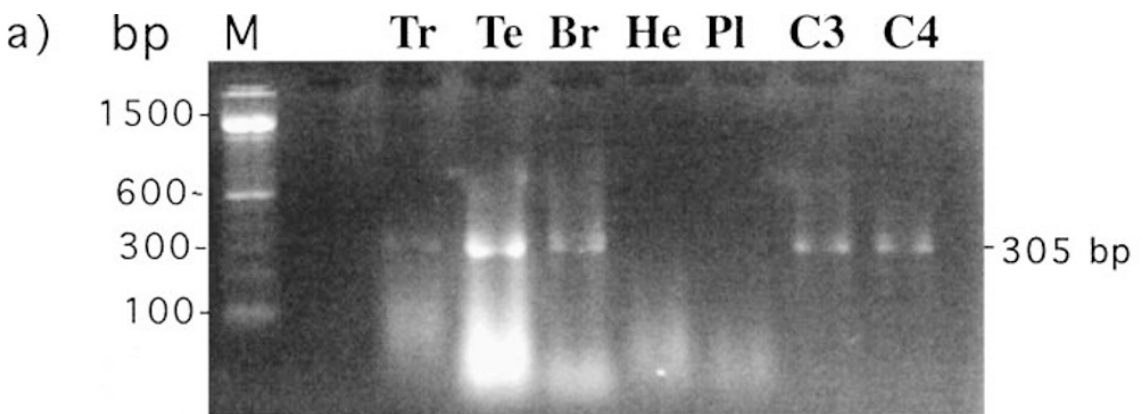

b)

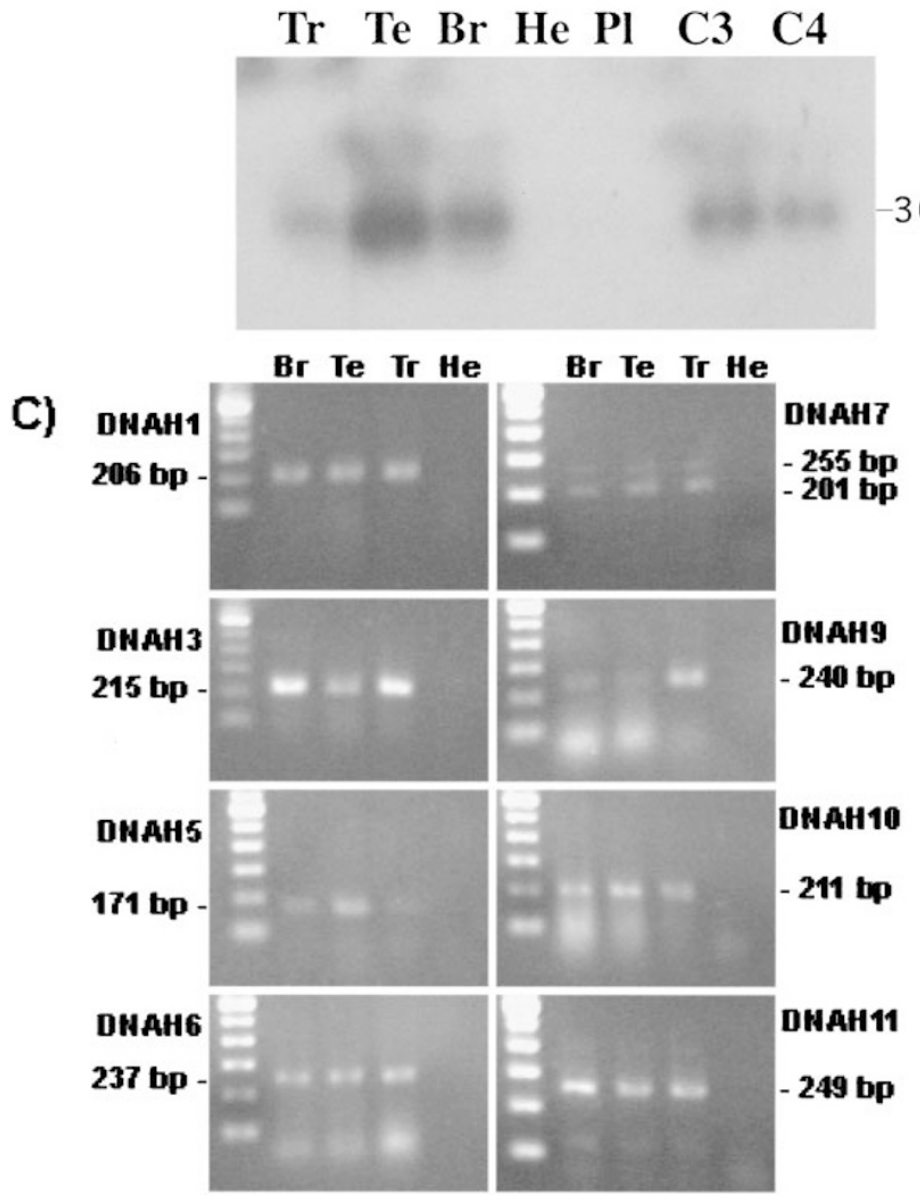

Figure 3 Expression of dynein heavy chain genes: a) RT-PCR is carried out with the axonema dynein specific DNAHloop-3F and DNAHloop-4R in different human tissue total RNAs. Lane M, 100 bp marker. Tr, Trachea; Te, Testis; Br, Brain; He, Heart; Pl, Placenta; C3 and C4, in vitro cultured ciliated epithelial cells with matured cilia. The major band which contains seven dynein isoforms (305 bp) is observed only in axonema containing tissue and ciliated epithelial cells but not in adult heart or placenta. b) The blot of this gel was hybridized with the pooled dynein probe and positive bands were observed only in axonema containing tissues,

c) Gene-specific primers (Table 1) were used to perform RT-PCR analysis of each dynein gene using RNA from brain (Br), testis (Te), trachea ( $\mathrm{Tr}$ ) and adult heart (He). Left lane: a 100 bp ladder. The expected amplification products were obtained except for

DNAH-7F/R which produced two different bands. These two bands ( 255 and $201 \mathrm{bp}$ ) were gel purified and sequenced. Note also that the $171 \mathrm{bp}$ band corresponds probably to both DNAH5 and DNAH8 since these isoform sequences were too identical in this region to design specific primers. 

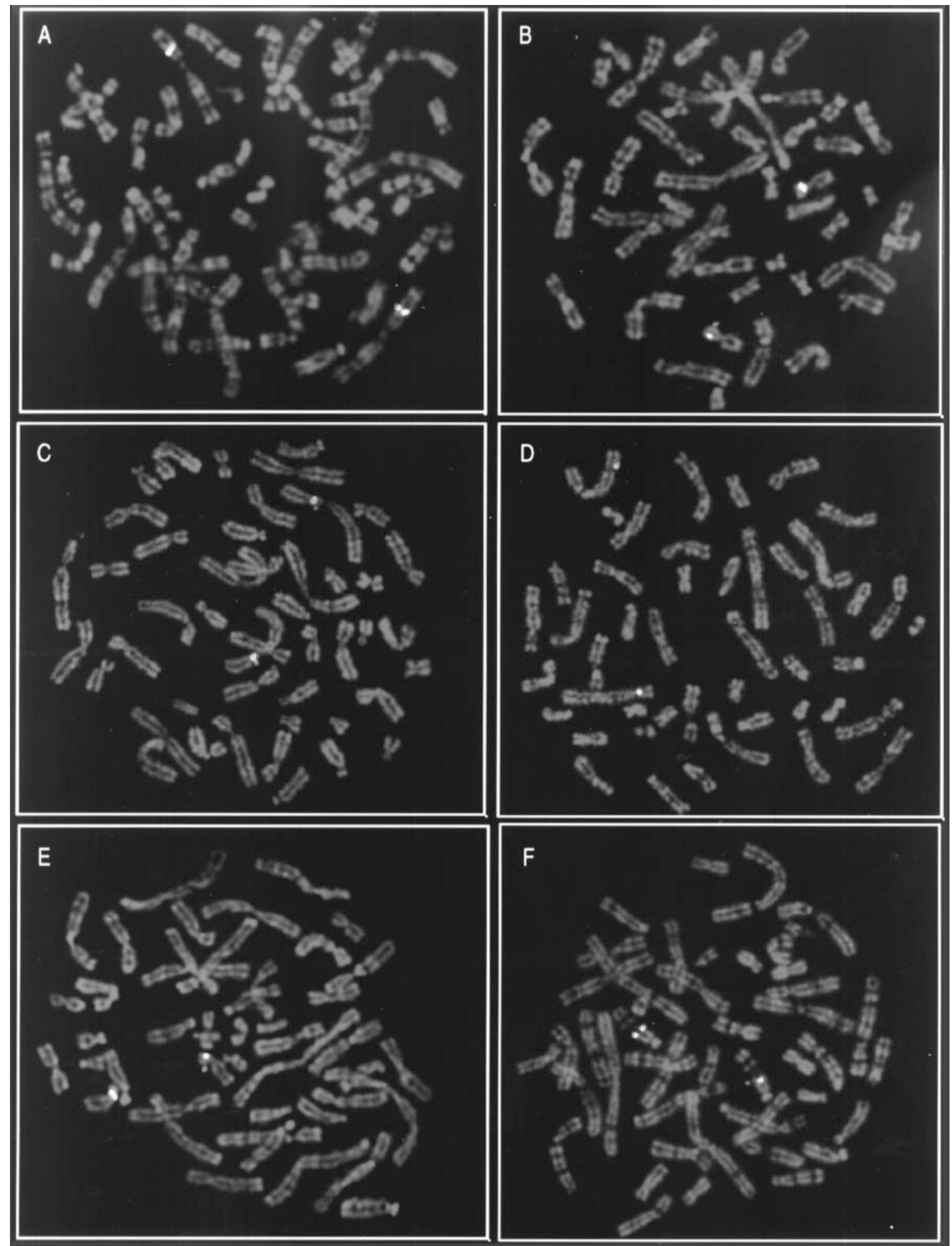

Figure 4 FISH mapping of PACs carrying the dynein heavy chain genes on human metaphase chromosomes. A PAC H2110

(DNAH1 gene), B PAC L1135 (DNAH3 gene), C PAC J1436 (DNAH6), D PAC P0764 (DNAH7p), E PAC P18162 (DNAH9), F PAC L2158 (DNAH10). 


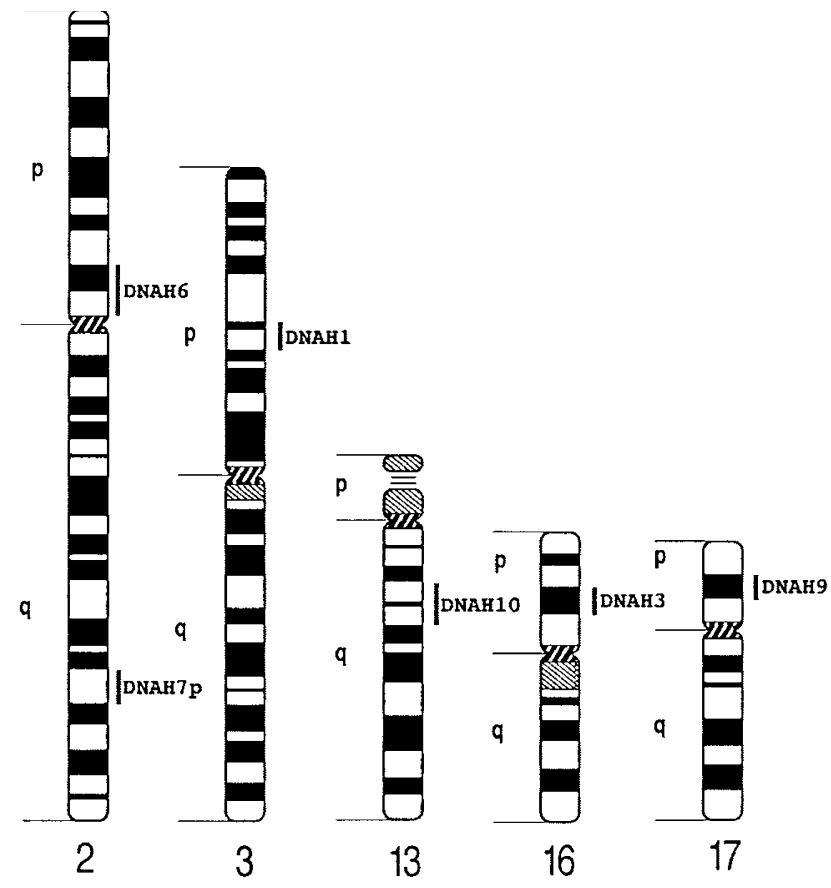

Figure 5 Chromosomal position of the dynein heavy chain genes are shown on the respective chromosome ideograms.

a normal spliced product, whereas the other ( $255 \mathrm{bp}$ ) was an un processed product. Since PAC clone P0764 amplified with the RT-DNAH7F and -7R primer pair a fragment with an A insertion before the normal GT donor site as the $255 \mathrm{bp}$ un processed product, we suspected that P0764 contains a DNAH7 pseudogene so that the human genome contains a DNAH7 gene and a pseudogene.

A rough quantitative estimation of dynein isoform transcription in ciliated epithelial cells could be deducted from the number of clones picked up during our screening. Among the seven dyneins that were amplified by DNAHloop-3F and DNAHloop-4R, DNAH1 and DNAH7, 2 putative inner arm dyneins and DNAH9, a putative outer arm dynein were the most abundant isoforms in ciliated cells of upper airways of the human body.

\section{Chromosomal localisation of dynein heavy chain genes} In order to map human dynein heavy chain genes, we screened the PAC human genomic library on filters obtained from Lawrence Livermore National Library (LLNL) with a 'pooled dynein probe' composed of the seven dynein cDNAs. We obtained 30 positive PACs that were scrutinised for dynein heavy chain isoform content by

(1) hybridisation with dynein heavy chain probe,

(2) PCR amplification with isoform specific primers and

(3) PAC DNA restriction digestion, Southern blotting, and hybridisation with pooled dynein probe.

PCR products and dynein positive bands were cloned and partially sequenced. Chromosomal assignment of selected PAC clones was done by fluorescent in situ hybridisation: DNAH1, which was present in five PACs maps to 3p21.2-p21.1 (Figure 4) as expected by the syntenic region of mouse. ${ }^{13,14}$ DNAH3 - the homologue of rat DLP3 - (11) maps to $16 \mathrm{p} 12$. DNAH6 containing PACs maps to 2p12-p11. DNAH10 containing PACs maps to 13q14. DNAH9 maps to $17 p 12$ and the pseudogene of DNAH7 to 2q33. Figure 5 summarises DNAH gene positions on chromosome ideograms.

FISH mapping was confirmed with primers amplifying from each dynein isoform (Table 3) except for DNAH9 which was independently mapped by FISH and radiation hybrid to $17 \mathrm{p} 12$ by Bartoloni et al. ${ }^{26}$

\section{Discussion}

We report here the isolation of dynein heavy chain genes from ciliated epithelium after in vitro ciliogenesis of the upper respiratory tract. Although the assembled dyneins are one of the major components for ciliary assembly and motility, it is unclear how many dynein heavy chain genes are involved in the process. We cloned here nine dynein heavy chain genes and their occurrence in axonema-containing tissues suggests that they are an integral part of the ciliary assembly or motility. In the course of this study, several groups reported the identification of dynein heavy chain genes from different human tissues such as lung, ${ }^{14}$ testis ${ }^{13}$ and genomic DNA. ${ }^{16}$ Comparing these sequences with ours, a new isoform has been isolated: (HSA)DNAH10, the homologue of which was al ready isolated in rat $^{11}$ but not from human testis and lung. According to the phylogenic tree, (HSA)DNAH10 is presumably a component of the inner dynein arm. The precise number of axonemal dynein heavy chain genes in mammals

Table 3 Results of RhyME analysis for mapping DNAH with radiation hybrids

\begin{tabular}{|c|c|c|c|c|c|c|c|}
\hline Dynein & AFM marker & D number & Lod score & Theta & $\begin{array}{l}\text { Distance } \\
\text { (CentiRad) }\end{array}$ & Map position & $\begin{array}{l}\text { PAC by } \\
\text { FISH }\end{array}$ \\
\hline DNAH1 & AFM287yd9 & D3S1588 & 3.17 & 0.572 & 314.1 & 3p21.2-p21.1 & 3p21.2-p21.1 \\
\hline DNAH3 & AFM $220 \times b 10$ & D16S417 & 4.65 & 0.491 & 299.9 & $16 p 12.3$ & $16 p 12$ \\
\hline DNAH6 & AFM 333vh5 & D2S388 & 11.07 & 0.263 & 562.3 & 2p12-p11.2 & 2p12-p11 \\
\hline DNAH7p $p^{a}$ & AFM 058ye3 & D2S115 & 5.13 & 0.407 & 1173.0 & $2 q 32-q 33$ & $2 q 33$ \\
\hline DNAHIO & AFM210wa5 & D13S168 & 6.43 & 0.357 & 322.2 & $13 q 14.3$ & $13 q 14$ \\
\hline
\end{tabular}

${ }^{a} p=p$ seudogene 
and in human is not known but there is a human homologue to each sea urchin isoform suggesting that all human axonemal isoforms have been cloned if (TGR)DNAH3C and (TGR)DNAH7D are considered as al ternative spliced CDNAs. Apart from two cytoplasmic dynein heavy chain genes DNCH1 and DNCH2 (both have P-loop signature TGKTESV $^{7,12}$ ) and the ruling out of Dnahc13, ${ }^{14,16}$ altogether 12 putative axonemal dynein heavy chain genes (with P-loop TGKTETT) have been isolated from human (DNAH1, DNAH2, DNAH3, DNAH5, DNAH6, DNAH7, DNAH8, DNAH9, DNAH10, DNAH11, DNAH12 and DNAH14). In phylogenic analysis, cytoplasmic and axonemal dynein heavy chains are distinctly separate and remain their nearest neighbours. DNAH11, DNAH9, DNAH5 and DNAH8 and their respective homologues in other organisms are considered to be potential outer arm dynein heavy chain genes. Similarly, DNAH1, DNAH2, DNAH3, DNAH6, DNAH7, DNAH10, DNAH12 and DNAH14 and their homologues are regarded as potential inner arm dynein heavy chain genes. Among these DNAH 12, which has also been isolated ${ }^{15}$ from HELA cells, a nonaxonema containing tissue could be taking part in organellar transport and ciliary assembly as speculated for DHC1B [(RNO) Dnah4), (RNO)Dnhc2] in rat. ${ }^{11,25}$

In our experiments, we were unable to clone DNAH5 and DNAH 8 cDNA fragments by common degenerated primers and ultimately cloned them by specific primers. The amino acid sequence of DNAH8 in the downstream common primer region is FITMNPG in human ${ }^{13}$ as in other dynein heavy chain genes but different in mouse and rat: FLTMNPG. ${ }^{11}$ By contrast the primer region sequence of DNAH5 has not been isolated in human but isoform 5 in mouse and rat is FLTMNPG. It is possible that the correct human sequence of these two genes is FLTMNPG instead of FITM NPG which may prevent transcription and subsequent amplification of these genes with the degenerated primer we selected (DNAHIoop$4 \mathrm{R})$.

Chromosomal assignment of these dynein genes is important for the pathogen icity of primary ciliary dyskinesia (PCD) in which ultrastructural abnormalities of inner and outer dynein arm is frequent. DNAH1 is mapped as expected to 3p21.2-p21.1, but DNAH12 which was also mapped to chromosome $3^{16}$ by somatic cell hybrids and was predicted to be mapped to this region by mouse synteny, ${ }^{14}$ was not evidenced in the five PACs hybridising to 3p21.2-p21.1. However, we cannot rule out that DNAH1 and DNAH12 are actually clustered and that we were unable to identify DNAH12 in the PAC clones containing DNAH1. By mouse synteny, DNAH 6 was predicted in two regions, 2p12-p11 and $6 \mathrm{p} 21.3^{14}$ but was mapped only to the $2 \mathrm{p} 12$ region (Figure 5).

\section{Acknowledgements}

The authors thank S Debrus for helpful discussions, B Duriez for unpublished information and fruitful comments, T Willems and $S$ Deckers for cell culture and D Depetris for excellent FISH preparations.
$M$ Gouy is kindly thanked for helping us to use SEAVIEW and PHYLO_W IN, prepare the data and get the phylogenetic trees. AKM was supported by a postdoctoral fellowship of the European Union (BIOMED2). This work was supported by a grant from the European Union (Biomed2, BMH4 CT96-0302). Sequences in this manuscript are submitted to EMBL database within accession numbers AJ132083 to AJ132094.

\section{References}

1 Dutcher KS: Flagellar assembly in two hundred and fifty easy to follow steps. TIG 1995; 11: 398-404.

2 Afzelius BA: A human syndrome caused by immotile cilia. Science 1976; 193: 317-319.

3 Asai DJ, Brokaw J: Dynein heavy chain isoforms and axonemal motility. Trends Cell Biol 1993; 3: 398-402.

4 Mitchell DR, Kang Y: Reversion analysis of dynein intermediate chain function. J Cell Sci 1993; 105: 1069-1078.

5 Le Dizet M, Piperno G: ida4-1, ida4-2 and ida4-3 are intron splicing mutations affecting the locus encoding p28, a light chain of Chlamydomonas axonemal inner dynein arms. Mol Biol Cell 1995; 6: 713-723.

6 Pennarun G, Escudier E, Chapelin C et al: Loss-of-function mutation in a human gene related to Chlamydomonas reinhardtii dynein IC78 result in primary ciliary dyskinesia. Am J Hum Genet 1999; 65: 1508-1519.

7 Asai DJ, Beckwith KA, Kandl KA, Keating HH, Tjandra H, Forney JD: The dynein heavy chain genes of Paramecium tetraaurelia. Sequences adjacent to the catalytic site P-loop identify cytoplasmic and axonemal heavy chain isoforms. J Cell Sci 1994; 107: 839-847.

8 Kamiya R: Mutation in 12 independent loci result in absence of outer dynein arms in Chlamydomonas reinhardtii. J Cell Biol 1988; 107: 2253-2258.

9 Porter ME, KnottJA, Myster SH, Farlow SJ: The dynein gene family in Chlamydomonas reinhardtii. Genetics 1996; 144: 569-585.

10 Gibbons BH, Asai DJ, Tang WJ, Hays TS, Gibbons IR: Phylogeny and expression of axonemal and cytoplasmic dynein genes in sea urchin. Mol Biol Cell 1994; 5: 136-155.

11 Tanaka Y, Zhang Z, Hirokawa N: Identification and molecular evolution of new dynein like protein sequences in rat brain. J Cell Sci 1995; 108: 1883-1893.

12 Andrews KL, Nettesheim P, Asai DJ, Ostrowsky LE: Identification of seven rat axonemal dynein heavy chain genes: Expression during ciliated cell differentiation. Mol Biol Cell 1996; 7: 71-79.

13 Neesen J, Koehler MR, Kirschner R et al: Identification of dynein heavy chain genes expressed in human and mouse testis: chromosomal localization of an axonemal dynein heavy chain gene. Gene 1997; 200: 193-202.

14 Vaughan KT, Mikami A, Paschal BM et al: Multiple mouse chromosomal loci for dynein based motility. Genomics 1996; 36: 29-38.

15 Vaisberg EA, Grissom PM, McIntosh JR: Mammalian cells express three distinct dynein heavy chains that are localized to different cytoplasmic organelles. J Cell Biol 1996; 133: 831-842.

16 Chapelin C, Duriez B, Magnino F, Goosens M, Escudier E, Amselem S: Isolation of several human axonemal dynein heavy chain genes: genomic structure of the catalytic site, phylogenetic analysis and chromosomal assignment. FEBS Lett 1997; 412: 325-330.

17 Jorissen M, van der Schueren B, den Berghe V, Cassiman JJ: The preservation and regeneration of cilia on human nasal epithelial cells cultured in vitro. Oto-Rhino-Laryngology 1989; 246: 308-314.

18 Jorissen $M$, Bessems A: Normal ciliary beat frequency after ciliogenesis in nasal epithelial cells cultured sequentially as monolayer and in suspension. Acta Otolaryngology 1995; 115: 66-70. 
19 Chomszynsky P, Sacchi N: Single step method of RNA isolation by acid guanidinium thiocyanate-phenol-chloroform extraction. Anal Biochem 1987; 162: 156-159.

20 Ausubel FM, Brent R, Kingston RE et al: Current Protocols in Molecular Biology. John Wiley and Sons: New York, 1987.

21 Galtier N, Gouy M, Gautier C: Seaview and Phylo_win: two graphic tools for sequence alignment and molecular phylogeny. CABIOS 1996; 12: 543-548.

22 Zehetner G, Lehrach H: The reference library system - sharing biological material and experimental data. Nature 1994; 367: 489-491.

23 Pinkel D, Straume T, Gray JW: Cytogenetic analysis using quantitative, high sensitivity, fluorescence hybridization. Proc Natl Acad Sci USA 1986; 83: 2934-2938.
24 Lemieux N, Dutrillaux B, Viegas-pequignot E: A simple method for simultaneous R-or G-banding and fluorescence in situ hybridization of single copy genes. Cytogenet Cell Genet 1992; 59: 311-312.

25 Chriswell PS, Ostrowsky LE, Asai DJ: A novel cytoplasmic dynein heavy chain: Expression of DYH1B in mammalian ciliated epithelial cells. J Cell Sci 1996; 109: 1891-1898.

26 Bartoloni L, Blouin J, Sainsbury AJ et al: Assignment of the human dynein heavy chain gene DNAH17L to human chromosome $17 p 12$ by in situ hybridization and radiation hybrid mapping. Cytogenet Cell Genet 1999; 84: 188-189. 